\title{
EDITORIAL
}

\section{What Can Semiconductor Fabs Do for the Green EARTH?}

Energy consumption for semiconductor factories has always been a sore point for environmental conservation. A study conducted by the European Union indicates that manufacturing a car generates less waste and uses fewer resources than manufacturing a laptop computer. Eight hundred kilowatt hours of electricity is used to manufacture one 200-mm semiconductor wafer, according to a topical report compiled by the Pacific Northwest Pollution Prevention Resource Center in 1999. Ironically, energy is cheap. The cost in Taiwan per kilowatt hour is about 3.6 NTD corresponding to US $\$ 0.12$, granted that the price is dictated by politics and may be raised momentarily to reflect the actual cost. The total energy cost to produce one $200-\mathrm{mm}$ wafer is US\$96 according to the price given here, while that wafer can sell for thousands of dollars according to the report. The situation of 300-mm wafers in 2008 is similar.

Raw input energy for a $300-\mathrm{mm}$ wafer from an ArF immersion scanner running at $150 \mathrm{wph}$ is about $1.8 \mathrm{~kW} \mathrm{~h}$ assuming that the scanner needs $165 \mathrm{~kW}$ of power. An EUV scanner is projected to require $640 \mathrm{~kW}$ for $100 \mathrm{wph}$ by the manufacturer, assuming a resist sensitivity of $10 \mathrm{~mJ} / \mathrm{cm}^{2}$. The raw energy for exposure per wafer is $10.4 \mathrm{~kW} \mathrm{~h}$ accordingly. Using resist sensitivity three times higher to achieve the bare minimum required line width roughness raises the raw energy to $31.2 \mathrm{~kW} \mathrm{~h}$. When the collector efficiency is $2 \%$ instead of $7.5 \%$ and Sn conversion efficiency is $2 \%$ instead of $5 \%$, the raw energy jumps to $293 \mathrm{~kW}$ h. The raw energy cost to expose a wafer at these four scenarios is $\$ 0.21, \$ 1.21, \$ 3.62$, and $\$ 34$, respectively. For 20 critical layers, the raw energy cost per wafer is $\$ 4.20, \$ 24.20, \$ 72.40$, and $\$ 680$, respectively. The cost in the last two cases starts to be a problem. Given that the assumption of the last case can be pessimistic, the realistic raw energy cost for exposure per wafer will probably be between $\$ 100$ and $\$ 200$.

What is the impact on the green earth? For arguments sake, if we use $800 \mathrm{~kW} \mathrm{~h}$ per wafer and scale it up to a 40,000-wafer-per-month fab, the fab needs $43.8 \mathrm{MW}$. To expose these wafers for 20 critical layers at $100 \mathrm{wph}$ requires 18 exposure tools and raw power for exposure, Il.6, 34.8, or $326 \mathrm{MW}$ depending on the pessimism for the technology. The factory needs to add $26 \%, 79 \%$, and $743 \%$ power capacity, respectively. The realistic scenario is probably doubling or tripling the factory power. The answer for a green-earth-conscientious fab is obvious.

Happy reading!

\section{Burn J. Lin Editor-in-Chief}

